\title{
Electrostatic Theory of Elementary Particles
}

\author{
Alexander Ivanchin \\ Orpheus Ltd., Tomsk, Russia \\ Email: alex_ivanchin@mail.ru
}

How to cite this paper: Ivanchin, A. (2017) Electrostatic Theory of Elementary Particles. World Journal of Nuclear Science and Technology, 7, 232-251.

https://doi.org/10.4236/wjnst.2017.74019

Received: July 2, 2017

Accepted: August 12, 2017

Published: August 15, 2017

Copyright $\odot 2017$ by author and Scientific Research Publishing Inc. This work is licensed under the Creative Commons Attribution International License (CC BY 4.0).

http://creativecommons.org/licenses/by/4.0/

\section{(c) (i) Open Access}

\begin{abstract}
Theoretical physics makes a wide use of differential equations for which only a potential solution is applied. The possibility that these equations may have a non-potential solution is ruled out and not considered. In this paper an exact non-potential solution of the continuity equation is described. The electric field of an elementary charged particle consists of two components: the known Potential Component (PC) produced by the charge and the earlier unknown Non-potential Component (NC) with a zero charge. Charged particles have both components, while a neutron has only the NC. The proton and neutron NC ensures similarity of their properties. The PC is spherically symmetric and NC is axisymmetric. Therefore, to describe an elementary particle, one should take into account both its spatial coordinates and the NC orientation. The particle interaction is determined by their NC mutual orientation. Neglecting the latter leads to indefiniteness of the interaction result. In a homogeneous electric field, the force acting on the NC is zero. Therefore, a charged particle possessing the NC will behave like a potential one. In an inhomogeneous field, the situation is principally different. Due to the NC there occurs an interaction between a neutron and a proton. The non-potential field results in the existence of two types of neutrons: a neutron and an antineutron. A neutron repels from a proton ensuring scattering of neutrons on protons. An antineutron is attracted to a proton leading to its annihilation. The NC produces the magnetic dipole moment of an elementary particle.
\end{abstract}

\section{Keywords}

Electrostatic Field of the Elementary Particles, Neutron Electrostatic Field, Neutron-Proton Interaction, Magnetic Moment of Elementary Particles, Antineutron-Proton Interaction

\section{Introduction}

In theoretical physics, not only the solution of differential equations is impor- 
tant, but the number of solutions as well. In quantum mechanics, as an example, boundary problems normally have several solutions and all these solutions are used to describe the behavior of an object. Discarding any of the solutions excludes one or another effect from consideration, and the description becomes inadequate and contrary to the fact. If a boundary problem has several solutions and only one of them is chosen, we should justify why we do so. For practically important boundary problems, the question of solution uniqueness as such is often not posed. It is thought that any obtained solution is automatically unique in accordance with the Cauchy-Kovalevsky theorem [1]; however, the theorem considers uniqueness for a problem only at a certain point and not over the entire domain. Proofs of uniqueness are provided for particular differential equations, e.g., two-dimensional potential boundary problems. However, conditions of the uniqueness theorem, e.g. boundedness of a solution or problem domain, may contradict actual physical phenomena.

In this work, using the field of a concentrated charge (electron, proton, neutron) as an example, it is shown that the well-known equation of mathematical physics has the second, earlier unknown solution. Taking into account this solution radically alters the interpretation of some physical processes. Surely, uniqueness of the solutions of differential equations is studied by mathematicians [1] [2], but their interest (and this is quite natural) is in mathematical rather than physical problems. The solution uniqueness of the electron problem has not been studied so far.

\section{Non-Potential Solution}

The electrostatic field of a point source obeys the equation

$$
\begin{gathered}
\nabla \cdot \boldsymbol{E}=-\frac{e}{4 \pi \varepsilon} \delta(\boldsymbol{r}) \\
\boldsymbol{E} \rightarrow 0, \quad \boldsymbol{r} \rightarrow \infty
\end{gathered}
$$

where $\boldsymbol{E}$ is the electrostatic field, $e$ is the electron charge, $\varepsilon$ is the dielectric constant, $\boldsymbol{r}$ is the radius-vector, and $\delta(\boldsymbol{r})$ is the delta (Dirac) function. It is assumed that $\boldsymbol{E}$ is a potential vector with the potential function $\Phi$ :

$$
\boldsymbol{E}=\nabla \cdot \Phi
$$

Equations (1) and (3) give the Poisson equation ( $\Delta$ is the Laplacian):

$$
\Delta(\Phi)=-\frac{e}{4 \pi \varepsilon} \delta(r)
$$

The solution of (4) is the following

$$
\Phi=\frac{e}{4 \pi \varepsilon r}
$$

In the Cartesian and spherical coordinates, the field vector $\boldsymbol{E}$ is written as

$$
\boldsymbol{E}=\frac{e}{4 \pi \varepsilon r^{3}} \boldsymbol{r}=\frac{e}{4 \pi \varepsilon}\left\langle\frac{1}{r^{2}}, 0,0\right\rangle
$$


In this work I will use pointed brackets to denote the vector component in the spherical coordinates. The sequence of the vector components in these brackets is as follows: radial, zenith, azimuth, e.g. $\left\langle E_{r}, E_{\vartheta}, E_{\varphi}\right\rangle$. The Cartesian components will be denoted by the braces, as it is accepted.

If two potential charges are located at a distance of $2 a$, then the energy of their interaction is written, according to (5), as

$$
W_{i}=\frac{e^{2}}{8 \pi \varepsilon a}
$$

The spherical and Cartesian coordinates are related as

$$
x=r \sin \vartheta \cos \varphi, \quad y=r \sin \vartheta \sin \varphi, \quad z=r \cos \vartheta
$$

The flux of the vector $\varepsilon \boldsymbol{E}$ through a closed sphere centered at the origin of the coordinates

$$
\varepsilon \oint_{S} E_{r} \mathrm{~d} S=\frac{e}{4 \pi \varepsilon} \int_{0}^{2 \pi} \mathrm{d} \varphi \int_{0}^{\pi} E_{r} r^{2} \sin \vartheta \mathrm{d} \vartheta=e
$$

The assumption about field potentiality (3) has no physical grounds and limits the class of the functions that can be a solution of (1). Hence, one needed to prove that (6) is a unique solution of Equation (1), but this was not done. If there is any other solution of (1), it is necessary to justify the choice of (6) as a physically feasible solution.

Let us find a different solution of (1) than the potential solution (6) by the procedure described in [3] [4] [5]. The auxiliary vector has the form

$$
\boldsymbol{U}^{+}=\left\{\frac{x}{r^{3}}, \frac{y}{r^{3}}, 0\right\}
$$

For the vector $\boldsymbol{U}^{+}$

$$
\nabla \cdot\left(\boldsymbol{U}^{+}\right)=\frac{1-3 \cos ^{2} \vartheta}{r^{3}}
$$

The vector $\boldsymbol{U}^{+}$is neither purely potential nor purely vortex because its divergence and rotor are not identically equal to zero. If we manage to find a solution for the equation

$$
\begin{aligned}
\Delta \Psi & =\frac{1}{r^{2}} \frac{\partial}{\partial r}\left(r^{2} \frac{\partial \Psi}{\partial r}\right)+\frac{1}{r^{2} \sin \vartheta} \frac{\partial}{\partial \vartheta}\left(\sin \vartheta \frac{\partial \Psi}{\partial \vartheta}\right)+\frac{1}{r^{2} \sin ^{2} \vartheta} \frac{\partial^{2} \Psi}{\partial \varphi^{2}} \\
& =\frac{1-3 \cos ^{2} \vartheta}{r^{3}}
\end{aligned}
$$

the vector

$$
\boldsymbol{U}=C\left(\boldsymbol{U}^{+}-\nabla \Psi\right)
$$

is the solution of equation [1], where $C$ is an arbitrary constant. One can replace without sacrifice of generality

$$
\frac{e}{2 \pi \varepsilon} C \rightarrow C
$$

Equation (12) is solved by the variable separation method 


$$
\Psi=R(r) \Theta(\vartheta) \Phi(\varphi)
$$

The function $\Psi$ must depend on $r, \vartheta$ and $\varphi$, one can use $R(r)=1 / r$ and the first term in (12) vanishes. The right side of (12) is invariant with $\varphi$. Let $\Phi(\varphi)=$ const . Hence, the third term in (12) vanishes and (12) takes the form

$$
\frac{\mathrm{d}}{\mathrm{d} \vartheta}\left(\sin \vartheta \frac{\mathrm{d} \Theta}{\mathrm{d} \vartheta}\right)=\left(1-3 \cos ^{2} \vartheta\right) \sin \vartheta
$$

Its integration with respect to $\vartheta$ gives:

$$
\frac{\mathrm{d} \Theta}{\mathrm{d} \vartheta}=-\sin \vartheta \cos \vartheta+\frac{A}{\sin \vartheta}
$$

where $A$ is an arbitrary constant, $-\infty<A<+\infty$. Further integration yields

$$
\Theta=\frac{1}{2} \cos ^{2} \vartheta+A \ln \tan \frac{\vartheta}{2}
$$

Although $\Theta$ is an unbounded function at $\vartheta=0$, the field vector $\nabla \Theta$ produced by the function is bounded at this point. It can be shown (which is done at the end of Section 2) that one of the condition of the minimum energy is $A=0$, and hence the second term in (18) is excluded from consideration. As a result, we have $\Theta=\left(\cos ^{2} \vartheta\right) / 2$ or in the Cartesian coordinates

$$
\Theta=\frac{z^{2}}{2\left(x^{2}+y^{2}+z^{2}\right)}
$$

From the above relation and from (15) it follows that

$$
\Psi=\frac{z^{2}}{2\left(x^{2}+y^{2}+z^{2}\right)^{3 / 2}}
$$

and from (13) one obtains

$$
\boldsymbol{U}=\frac{e}{4 \pi \varepsilon} \frac{z^{2}-2 x^{2}-2 y^{2}}{\left(x^{2}+y^{2}+z^{2}\right)^{5 / 2}} \boldsymbol{r}=\frac{e}{4 \pi \varepsilon r^{2}}\left\langle 2-3 \cos ^{2} \vartheta, 0,0\right\rangle
$$

Here $C=1$ is selected to fulfill (22). Substitution of (21) in (1) proves that (21) is a solution of (1). The vector $\boldsymbol{U}$ is nonpotential, since $\nabla \times \boldsymbol{U} \equiv 0$.

The vector $\boldsymbol{U}$ is axisymmetric about the $z$ axis, since $U_{r}$ does not depend on $\varphi$. The flux of the vector $\boldsymbol{U}$ through a closed sphere centered at the origin of the coordinates is

$$
\varepsilon \oint_{S} U_{r} \mathrm{~d} S=\frac{e}{4 \pi \varepsilon} \int_{0}^{2 \pi} \mathrm{d} \varphi \int_{0}^{\pi} U_{r} r^{2} \sin \vartheta \mathrm{d} \vartheta=-e
$$

The flux (22) is constant and is independent of the sphere radius, which suggests that

$$
\nabla \cdot \boldsymbol{U}=-\frac{e}{4 \pi \varepsilon} \delta(r)
$$

as is for $\boldsymbol{E}$ in (6). From (6) and (21) it follows that the vectors $\boldsymbol{E}$ and $\boldsymbol{U}$ are parallel. 


\section{Energy}

The vectors $\boldsymbol{U}$ and $\boldsymbol{E}$ satisfy the boundary problem (1). Consider the linear combination of the vectors

$$
\lambda_{1} \boldsymbol{E}+\lambda_{2} \boldsymbol{U}
$$

where $\lambda_{1}, \lambda_{2}$ are the arbitrary constants. The choice of the constants may not affect the charge and the charge must remain constant and equal to the electron charge.

The charge value produced by the fields (6) and (21) is determined by the relations (9) and (22). If the values of the parameters $\lambda_{1}$ and $\lambda_{2}$ are selected in this way

$$
\lambda_{1}=\lambda_{2}=\lambda_{n}
$$

where $\lambda_{n}$ is the arbitrary parameter, then the electric field will be represented like this

$$
\boldsymbol{N}=\lambda_{n}(\boldsymbol{U}+\boldsymbol{E})=\lambda_{n} \frac{3 e\left(2 z^{2}-x^{2}-y^{2}\right)}{4 \pi \varepsilon r^{5}} \boldsymbol{r}=\lambda_{n} \frac{e}{8 \pi \varepsilon}\left\langle\frac{1+3 \cos 2 \vartheta}{r^{2}}, 0,0\right\rangle
$$

The vector flux $N$ in (30) through the sphere with the center at the origin of the coordinates

$$
\iint_{S} N_{r} d S=\lambda_{N} \frac{e}{8 \pi \varepsilon} \int_{0}^{\pi} \mathrm{d} \vartheta \int_{0}^{2 \pi}(1+3 \cos 2 \vartheta) \mathrm{d} \varphi=0
$$

is zero, which means that the electric field (26) is produced by a zero charge. This is the property of the nonpotential field, whereas it is impossible in the case of the classical potential field. The field (26) at a certain value of $\lambda_{n}$ describes the neutron electric field (see the details in Section 4), therefore, I will refer to the value $N$ in (26) as a neutron component, and the parameter $\lambda_{n}$-a neutron charge. In the spherical coordinates (26), the field has only one radial component independent of the azimuthal angle $\varphi$, which means that the neutron field is axisymmetric, with the applicate, in this case, being the axis of symmetry. In the general case, the orientation of the neutron field will be specified if the direction of the axis of symmetry is indicated.

If the potential field (6) is added (26), one will derive a general solution of the problem of the electron (proton) in the form

$$
\boldsymbol{T}=\boldsymbol{E}+\boldsymbol{N}
$$

Indeed, the flux of the vector $\varepsilon \boldsymbol{T}$ through a closed surface is equal to $e$, i.e. the elementary charge, according to (9) and (27). The non potentiality of the electric field charge results in the appearance of a "free" component of the electric field.

The potential energy of the electrostatic field (24) is specified by the relation [6]

$$
W=\frac{\varepsilon}{2} \int_{V_{\rho}}|\boldsymbol{T}|^{2} \mathrm{~d} V=\frac{\varepsilon}{2} \int_{V_{\rho}}\left|\boldsymbol{E}+\lambda_{n} \boldsymbol{N}\right|^{2} \mathrm{~d} V
$$


Using (6) and (21), we obtain:

$$
W=\frac{e^{2}}{32 \pi^{2} \varepsilon_{V_{\rho}}} \int_{r}\left(E_{r}+\lambda_{n} N_{r}\right)^{2} \mathrm{~d} V
$$

The lower index $\rho$ by the integration limit $V_{\rho}$ means that the integration is performed with respect to the whole space with the exception of the sphere of the small radius $\rho$ with the center at the origin of the coordinates. This spherical environment has been excluded from consideration to avoid the integral divergence at the origin of the coordinates, $\rho$ is the radius of truncation and the environment $V_{\rho}$ will be referred to as a source to emphasize the fact that the electric field possessing potential and nonpotential components is its product. A particle can have both the potential and neutron component, each of them having its own radius of truncation. The integration is performed over the entire space. Relation (30) is broken down into three terms. The first term

$$
W_{P}=\frac{e^{2}}{32 \pi^{2} \varepsilon} \int_{V_{\rho}} E_{r}^{2} \mathrm{~d} V=\frac{e^{2}}{32 \pi^{2} \varepsilon} \int_{\rho}^{\infty} \frac{\mathrm{d} r}{r^{2}} \int_{0}^{2 \pi} \mathrm{d} \varphi \int_{0}^{\pi} \sin \vartheta \mathrm{d} \vartheta=\frac{e^{2}}{8 \pi \varepsilon \rho}
$$

is the self-energy for the classical potential electron component. The second term

$$
\begin{aligned}
W_{N} & =\lambda_{n}^{2} \frac{e^{2}}{32 \pi^{2} \varepsilon} \int_{V_{\rho_{n}}} N_{r}^{2} \mathrm{~d} V \\
& =\lambda_{n}^{2} \frac{e^{2}}{128 \pi^{2} \varepsilon} \int_{\rho_{n}}^{\infty} \frac{\mathrm{d} r}{r^{2}} \int_{0}^{2 \pi} \mathrm{d} \varphi \int_{0}^{\pi}(1+3 \cos 2 \vartheta)^{2} \sin \vartheta \mathrm{d} \vartheta \\
& =\frac{\lambda_{n}^{2} e^{2}}{10 \pi \varepsilon \rho_{n}}
\end{aligned}
$$

is the self-energy for the non potential component. The third term

$$
W_{I}=\lambda_{n} \frac{e^{2}}{16 \pi^{2} \varepsilon} \int_{V_{\rho}} E_{r} N_{r} \mathrm{~d} V=\lambda_{n} \frac{e^{2}}{16 \pi^{2} \varepsilon} \int_{\rho}^{\infty} r d r \int_{0}^{2 \pi} \mathrm{d} \varphi \int_{0}^{\pi} \frac{1+3 \cos 2 \vartheta}{r^{2}} \sin \vartheta \mathrm{d} \vartheta=0
$$

is the interaction energy for the potential and nonpotential components. Here $W_{P}$ is the potential component energy, $W_{N}$ is the nonpotential component energy, $W_{I}$ is the energy of the interaction between the potential and nonpotential components. Since $W_{I}=0$, there is no interaction between the potential and neutron components. The presence of the neutron component only increases the intrinsic energy of the particle.

The value of $\rho$ can be estimated equating the electrostatic energy of the particle to the Einstein energy $m c^{2}$, where $m$ is the particle mass and $c$ is the light speed. The existence of the neutron component as well as the potential one is due to the properties of the source. The radius of truncation for different components can be different, which the lower index $n$ by the radius does denote (32). For estimation let us assume the radius of truncation of the particle to be the same both of the potential and non potential components, since they are 
produced by the same source. Adding (31) and (32) and equating the sum to $m c^{2}$ one obtains

$$
W_{P}+W_{N}=\frac{e^{2}}{40 \pi \varepsilon \rho}\left(5+4 \lambda_{n}^{2}\right)=m c^{2}
$$

The value of the multiplier $\lambda_{n}$ is unknown. To estimate the truncation radius of a charged particle, one can assume $\lambda_{n}=0$, i.e. estimating the radius by the potential component one derives, as a result

$$
\rho=\frac{e^{2}}{8 \pi \varepsilon m c^{2}}
$$

As a matter of fact, the real radius of truncation will be slightly larger due to the energy of the neutron component. For a proton of the mass $m_{p}=1.6726218 \times 10^{-27} \mathrm{~kg}$ [7] gives $\rho_{p}=7.67311 \times 10^{-19} \mathrm{~m}$, for an electron with the mass $m_{e}=9.109383 \times 10^{-31} \mathrm{~kg}$ one obtains $\rho_{e}=1.4089 \times 10^{-15} \mathrm{~m}$. For a neutron instead of (34) one has

$$
\frac{\rho_{n}}{\lambda_{n}^{2}}=\frac{e^{2}}{10 \pi \varepsilon m_{n} c^{2}}=6.13003 \times 10^{-19} \mathrm{~m}
$$

Here, on the left-hand side of the equality there is unknown parameter $\lambda_{n}$, $m_{n}$ is the neutron mass. For a charged particle with the field (28) the parameters of the neutron component are determined by its mass, the magnetic and electric moment, each particle will have its own values of $\lambda_{n}$ and $\rho_{n}$. At present there are not enough experimental data for their unambiguous calculation.

In addition to the main component $\mathrm{T}$ in (28), the dipole and multipole components, whose fields are derived from (28) by differentiation with respect to the spatial coordinates, can contribute to the total field. These components can also be produced by a source just as the main components. For the multipole components the size of the sources can differ and their value will affect the total system energy.

Let explain why we put in (18) $A=0$. In spherical coordinates, the gradient of the second term in the right side of (18)

$$
\nabla\left[A \ln \tan \frac{\vartheta}{2}\right]=\frac{A}{r^{2}}\left\langle-\ln \tan \frac{\vartheta}{2}, \frac{1}{\sin \vartheta}, 0\right\rangle
$$

Then, the interaction energy for the classical component (6) and (37) is written as

$$
-\frac{A e}{4 \pi \varepsilon} \int_{\rho}^{\infty} \frac{\mathrm{d} r}{r^{2}} \int_{0}^{\pi} \ln \tan \frac{\vartheta}{2} \sin \vartheta \mathrm{d} \vartheta \int_{0}^{2 \pi} \mathrm{d} \varphi=0
$$

The interaction energy for the nonpotential component (21) and (37) is zero, too. Hence, the minimum energy (29) is reached with $A=0$.

\section{Neutron}

The formula for the neutron electric field (26) contains the arbitrary constant 
$\lambda_{n}$. There are two possible variants: $\lambda_{n}>0$ and $\lambda_{n}<0$. This agrees well with the known fact that there are two types of neutrons: a neutron and an antineutron. These are two different particles. The sign for $\lambda_{n}$ must be chosen proceeding from the interaction. A proton carries the positive charge, annihilates with an antineutron, they are attracted and, therefore, with the antineutron $\lambda_{n}<0$ and with the neutron $\lambda_{n}>0$.

An arbitrary rotation can be realized by three rotations about the coordinate axes. First, a rotation about the $z$ axis through the angle $\alpha$ is made. In this rotation, the $x$ and $y$ axes take the positions $x^{\prime}$ and $y^{\prime}$. The second rotation is made about the $y^{\prime}$ axis through the angle $\beta$ and the $x^{\prime}$ axis assumes the position $x^{\prime \prime}$. The third rotation is made about the $x^{\prime \prime}$ axis through the angle $\gamma$. For the charge at the origin of the coordinates, the initial orientation is chosen such that the symmetry axis coincides with the $z$ axis. In this case, rotation about the $z$ axis through any angle $\alpha$ changes nothing. Therefore, it can be assumed that $\alpha=0$. The matrices for the rotations about the $y$ and $x$ axes are written as [8]

$$
A_{y}=\left(\begin{array}{ccc}
\cos \beta & 0 & \sin \beta \\
0 & 1 & 0 \\
-\sin \beta & 0 & \cos \beta
\end{array}\right), \quad A_{x}=\left(\begin{array}{ccc}
1 & 0 & 0 \\
0 & \cos \gamma & -\sin \gamma \\
0 & \sin \gamma & \cos \gamma
\end{array}\right)
$$

The vectors are transformed using the matrices $A=A_{x} A_{y}$ and the coordinates using the transposed matrix $A^{\prime}$. Transformation of the vector $N$ in (26) gives

$$
N=\frac{\lambda_{n} e}{16 \pi \varepsilon} \frac{q}{r^{5}} r
$$

where

$$
\begin{aligned}
q= & 6\left(z^{2}-x^{2}\right) \cos 2 \beta \cos ^{2} \gamma+\left(x^{2}-2 y^{2}+z^{2}\right)(3 \cos \gamma-1) \\
& +12 x z \cos ^{2} \gamma \sin 2 \beta-12 y z \cos \beta \sin 2 \gamma-12 x y \sin \beta \sin 2 \gamma
\end{aligned}
$$

Because of the stress field symmetry, the angles are variable as follows

$$
0 \leq \beta<\pi, \quad 0 \leq \gamma<\pi
$$

\section{Interaction Energy}

Let us denote the external homogeneous electric field $\tilde{\boldsymbol{E}}=\left\{\tilde{E}_{x}, \tilde{E}_{y}, \tilde{E}_{z}\right\}$ by the tilde. It has an arbitrary value and orientation, and in the spherical coordinates its radial component will be written as

$$
\tilde{E}_{r}=\tilde{E}_{x} \sin \vartheta \cos \varphi+\tilde{E}_{y} \sin \vartheta \sin \varphi+\tilde{E}_{z} \cos \vartheta
$$

The other spherical components are of no interest, since only the radial component of the neutron component is other than zero. Here $\left\{\tilde{E}_{x}, \tilde{E}_{y}, \tilde{E}_{z}\right\}=$ const is the external field in the Cartesian coordinates. The interaction energy of the neutron component with the external field is

$$
\varepsilon \int_{V_{\rho}}(\boldsymbol{N} \cdot \tilde{\boldsymbol{E}}) \mathrm{d} V=0
$$


The interaction energy of a neutron and a homogeneous electric field is equal to zero. This means that the force acting on the neutron on the side of the homogeneous electric field is also equal to zero. The same equality may not be true for the nonuniform external field. That a neutron, under certain conditions, interacts with an electric field is an experimental fact [9]. The interaction energy of the particles with a homogeneous electric field is completely determined by the potential component. The non-potential component $N$ does not participate in the interaction. In a homogeneous electric field it is impossible to tell if an electron (a proton) possesses the non-potential component. It behaves like a particle with a purely potential field.

\subsection{Interaction of the Potential Proton and Neutron}

From (33) it follows that there is no interaction between the potential and neutron component of the same particle. However, if the particles are different, for instance, at the point on the axis of abscissa $x=a$ there is a particle with a pure potential field (6), and at the point $x=-a$ there is a particle with an arbitrary oriented neutron field (40), then between them there is interaction with the energy

$$
W_{e n}=\varepsilon \int_{V_{\rho}^{\prime}}\left(\boldsymbol{E}_{-} \cdot \boldsymbol{N}_{+}\right) \mathrm{d} V
$$

The lower indices - and + denote that the center of the given component is located on the axis of abscissa at the points $-a$ and $+a$, respectively. Integration is performed over the whole space with the exception of two spherical regions with the radii $\rho$ at the point $x=+a$ and

$$
k \rho
$$

at the point $x=-a$. Here it is taken into account that the particles can have different radii of truncation. The potential field of the electric charge of the particle at the point $x=-a$ is

$$
\boldsymbol{E}_{-}=\frac{e}{4 \pi \varepsilon r_{-}^{3}} \boldsymbol{r}_{-}
$$

and the neutron field at the point $x=a$ is

$$
\boldsymbol{N}_{+}=\frac{\lambda_{n} e}{16 \pi \varepsilon} \frac{q_{+}}{r_{+}^{5}} \boldsymbol{r}_{+}
$$

where

$$
\begin{aligned}
q_{+}= & 6\left[z^{2}-(x-a)^{2}\right] \cos 2 \beta \cos ^{2} \gamma+\left[(x-a)^{2}-2 y^{2}+z^{2}\right](3 \cos \gamma-1) \\
& +12(x-a) z \cos ^{2} \gamma \sin 2 \beta-12 y z \cos \beta \sin 2 \gamma-12(x-a) y \sin \beta \sin 2 \gamma
\end{aligned}
$$

Integration (45) is performed by passing to the bipolar coordinates, there is a scalar under the integral and the Cartesian coordinates are replaced by the bipolar ones, according to the formulas

$$
x=a \frac{\sinh \tau}{\cosh \tau-\cos \sigma}, \quad y=a \frac{\sin \sigma \cos \varphi}{\cosh \tau-\cos \sigma}, \quad z=a \frac{\sin \sigma \sin \varphi}{\cosh \tau-\cos \sigma}
$$


The Jacobian is

$$
Y=a^{3} \frac{\sin \sigma}{(\cosh \tau-\cos \sigma)^{3}}
$$

The field (48) is derived from (40) by the transformation of the shift along the axis of abscissa $x \rightarrow x-a$, and the field (47) from (6) by the shift to the opposite side $x \rightarrow x+a$. Here $r_{+}$and $r_{-}$are

$$
\begin{gathered}
r_{+}=\sqrt{(x-a)^{2}+y^{2}+z^{2}}=a \sqrt{2 \frac{\cosh -\sinh \tau}{\cosh \tau-\cos \sigma}} \\
\boldsymbol{r}_{+}=\{x-a, y, z\} \\
r_{-}=\sqrt{(x+a)^{2}+y^{2}+z^{2}}=a \sqrt{2 \frac{\cosh +\sinh \tau}{\cosh \tau-\cos \sigma}} \\
\boldsymbol{r}_{-}=\{x+a, y, z\}
\end{gathered}
$$

As a result, one obtains the interaction energy value (45) in the form

$$
\begin{aligned}
W_{e n}^{\prime}= & \varepsilon \int_{-(q+Q)}^{Q} \mathrm{~d} \tau \int_{0}^{\pi} \mathrm{d} \vartheta \int_{0}^{2 \pi}\left(\boldsymbol{E}_{-} \cdot \boldsymbol{N}_{+}\right) Y \mathrm{~d} \varphi \\
= & \frac{\lambda_{n} e^{2}}{3072 \pi \varepsilon a}\left(1+3 \cos 2 \beta-6 \cos 2 \gamma \sin ^{2} \beta\right) \exp [-3(q+Q)] \\
& \cdot\{-16 \exp [3(q+Q)] \ln [1-\exp 2 Q]+16 \exp [3(q+Q)] \\
& \ln (1-\exp [-2(q+Q)])-5[2 \exp (q+Q)[\exp (2 q+4 Q)-1] \\
& \left.+\ln \operatorname{coth} \frac{q+Q}{2}\right]+\exp [2(q+Q)][\exp q(5 \exp 4 Q-15-6 \exp 2 Q) \\
& \left.\left.\times \ln \operatorname{coth} \frac{Q}{2}+3[2+5 \exp 2(q+Q)] \ln \operatorname{coth} \frac{q+Q}{2}\right]\right\}
\end{aligned}
$$

Here

$$
Q=\ln \frac{2 a}{\rho}, \quad q=-\ln k
$$

$k$ is the coefficient from (46). The condition of $\rho \ll a$ is necessary to make the calculations correctly. This condition is fulfilled for real variants for which the value

$$
Q \sim 10 \div 20
$$

Using the above value $Q$ as well as an approximate representation for small values of $x$

$$
\ln (1+x) \approx x
$$

it is possible to simplify the Formula (56)

$$
W_{e n}^{\prime}=\frac{\lambda_{n} e^{2}}{1536 \pi \varepsilon a}\left(1+3 \cos 2 \beta-6 \cos 2 \gamma \sin ^{2} \beta\right)(9-16 Q)
$$

Thus, the energy of interaction between the neutron and the potential charge has a weak logarithmic dependence on the value of the truncation radius and is 
inversely proportional to the distance between the particles. It also depends on the neutron orientation and can have both positive and negative values, that is, the interaction of the potential charge with the neutron component can be both attraction and repulsion. The different value of the radii of truncation was taken into account in the initial Formula (45). However, the influence of their difference on the final result turned out negligible. Further, I am not going to take into consideration this difference.

If there is a neutron at the point $x=-a$ and a potential charge at the point $x=+a$, i.e. the particles exchange their places, then in (60) it is necessary to replace the angle $\beta$ by $\beta_{-}$. The transformation is performed according to (62), and the energy found

$W_{e n}^{\prime \prime}=\varepsilon \int_{-Q}^{Q} \mathrm{~d} \tau \int_{0}^{\pi} \mathrm{d} \vartheta \int_{0}^{2 \pi}\left(\boldsymbol{E}_{+} \cdot \boldsymbol{N}_{-}\right) Y \mathrm{~d} \varphi=\frac{\lambda_{n} e^{2}}{1536 \pi \varepsilon a}\left(1+3 \cos 2 \beta_{-}-6 \sin ^{2} \beta_{-}\right)(9-16 Q)(61)$

depends only on one angle $\beta_{-}$. This energy is needed when calculating the energy of the interaction of two charged particles, each of them having its own neutron component.

\subsection{Interaction of Two Arbitrary Oriented Neutrons}

It is always possible to choose the coordinate orientation in such a way that the symmetric axis of the charge located at the point $x=-a$ would lie in the $x Z$ plane. Due to the choice of the above orientation of the coordinate system, the angle $\gamma_{-}=0$, and this neutron orientation is determined only by the angle $\beta_{-}$. This choice of the orientation of the coordinate system does not decrease the generality of consideration. The neutron field at the point $x=-a$ is derived from (48) by the replacement

$$
a \rightarrow-a, \beta_{+} \rightarrow \beta_{-}, \gamma_{+} \rightarrow 0
$$

As a result, one obtains the expression for the neutron at the point $x=-a$ :

$$
\boldsymbol{N}_{-}=\frac{\lambda_{n} e}{8 \pi \varepsilon r_{-}^{5}}\left\{(x+a)^{2}-2 y^{2}+z^{2}+3\left[z^{2}-(x+a)^{2}\right] \cos 2 \beta_{-}+6(x+a) z \sin 2 \beta_{-}\right\} \boldsymbol{r}_{-}
$$

The interaction energy of the neutron-neutron will be written as

$$
\begin{aligned}
W_{n n}= & \varepsilon \int_{V_{\rho}^{\prime}}\left(N_{+} \cdot N_{-}\right) \mathrm{d} V=\varepsilon \int_{-Q}^{Q} \mathrm{~d} \tau \int_{0}^{\pi} \mathrm{d} \sigma \int_{0}^{2 \pi}\left(N_{+} \cdot N_{-}\right) Y \mathrm{~d} \varphi \\
= & \pm \lambda_{N}^{2} \frac{e^{2}}{4056 \pi \varepsilon a} \sinh Q\{336(3 \cos 2 \gamma-1) \cosh Q \\
& -48\left[\left(2 \cos 2 \beta-7 \cos 2\left(\beta-\beta_{-}\right)\right)-39 \cos 2\left(\beta+\beta_{-}\right)\right] \cos ^{2} \gamma \\
& +\cos 2 \beta_{-}(3 \cos 2 \gamma-1) \cosh Q+4 \ln \frac{1+\cosh Q}{1-\cosh Q}\left[-6 \cos 2 \beta\left(5+13 \cos 2 \beta_{-}\right)(64)\right. \\
& \times \cos ^{2} \gamma-\left(23+15 \cos 2 \beta_{-}\right)(3 \cos 2 \gamma-1)-3\left(-7-2 \cos 2 \beta \cos ^{2} \gamma\right. \\
& \left.+\cos 2 \beta_{-}\left(1+46 \cos 2 \beta \cos ^{2} \gamma-3 \cos 2 \gamma\right)+21 \cos 2 \gamma\right) \cosh 2 Q \\
& \left.\left.+768 \cos \beta \cos \beta_{-} \cos ^{2} \gamma \sin \beta \sin \beta_{-} \sinh ^{2} Q\right]\right\}
\end{aligned}
$$


Neutrons interact with each other due to the nonpotential electric field. The energy (64) depends both on the charge signs $\lambda_{n}$ and the mutual orientation. Depending on it the same neutrons can be both attracted and repulsed. At present there are no experimental data on the neutron-neutron interaction. The formula (64) for large values of $Q$ is simplified

$$
\begin{aligned}
W_{n n}= & \pm \frac{\lambda_{n}^{2} e^{2}}{512 \pi \varepsilon a}\left\{2-18 \cos 2 \beta-33 \cos 2\left(\beta-\beta_{-}\right)+18 \cos 2 \beta_{-}+63 \cos 2\left(\beta+\beta_{-}\right)\right. \\
& \left.-3 \cos 2 \gamma\left[2+6 \cos 2 \beta+2(9-5 \cos 2 \beta) \cos 2 \beta_{-}+32 \sin 2 \beta \sin 2 \beta_{-}\right]\right\}
\end{aligned}
$$

For instance, for the parameters $\beta=\beta_{-}=\gamma=0$ the interaction energy value will be such

$$
W_{n n}= \pm \lambda_{n}^{2} \frac{9 e^{2}}{512 \pi \varepsilon a}
$$

\subsection{Interaction Energy of Non-Potential Particles}

The obtained results allow defining the proton-proton interaction energy. The total energy of the field of two non-potential charged particles will be written, as

$$
\begin{aligned}
W= & W_{e e}+W_{n n}+W_{e n}^{\prime}+W_{e n}^{\prime \prime} \\
= & \frac{e^{2}}{1536 \pi \varepsilon a}\left\{192+\lambda_{n}\left(16 Q-9+6 \lambda_{n}\right)\right. \\
& +6 \lambda_{n} \cos 2 \beta_{-}\left(9-16 Q+9 \lambda_{n}+30 \lambda_{n} \cos 2 \beta \cos ^{2} \gamma-27 \lambda_{n} \cos 2 \gamma\right) \\
& -3 \lambda_{n}\left[2\left(16 Q-9+18 \lambda_{n}\right) \cos 2 \beta \cos ^{2} \gamma+\left(9-16 Q+6 \lambda_{n}\right) \cos 2 \gamma\right. \\
& \left.\left.+192 \lambda_{n} \cos ^{2} \gamma \sin 2 \beta \sin 2 \beta_{-}\right]\right\}
\end{aligned}
$$

Here (7), (60), (61), (65) are used as well as the assumption that the truncation radii of the potential and neutron components are the same.

\section{Potential and Non-Potential Magnetic Field}

A magnetic field like an electric field can have potential and non-potential components. To describe a potential magnetic field, the notion of the vector-potential [8] is used. For a point magnetic charge (a monopole) located at the origin of the coordinates and oriented along the $z$-axis, the vector-potential is written as

$$
\frac{\mu}{4 \pi}\left\{0,0, \frac{\chi}{r}\right\}
$$

$\mu$ is the magnetic constant, $\chi$ is the value of the monopole charge. Monopoles have not been found in reality, so it is not clear if they exist in nature. Here the notion of a monopole is used as a mathematical object to solve problems. Taking the rotor from (68), one obtains the field strength of the magnetic monopole in the form

$$
\boldsymbol{M}=\frac{\mu}{4 \pi} \nabla \times\left\{0,0, \frac{\chi}{r}\right\}=\frac{\mu \chi}{4 \pi r^{3}}\{-y, x, 0\}=\frac{\mu \chi}{4 \pi r^{2}}\langle 0,0, \sin \vartheta\rangle
$$

From here it is clear that in the spherical coordinates only the azimuthal 
component of the monopole strength is other than zero. Differentiating (68) with respect to $\mathrm{z}$ and replacing $\chi$ by the magnetic moment $p$, one obtains the vector-potential of the point magnetic dipole in the form

$$
\mu \frac{p}{4 \pi}\left\{0,0, \frac{z}{r^{3}}\right\}
$$

Magnetic dipoles really exist, for instance, the magnetic electron moment. The rotor from (70) is the magnetic field induction produced by the magnetic dipole.

$$
\boldsymbol{B}=\mu \frac{p}{4 \pi} \nabla \times\left\{0,0, \frac{z}{r^{3}}\right\}=\mu \frac{3 p}{4 \pi} \frac{z}{r^{5}}\{-y, x, 0\}=\frac{3 \mu p}{8 \pi r^{3}}\langle 0,0, \sin 2 \vartheta\rangle
$$

In the spherical coordinates only the azimuthal component is other than zero, it decreases with a distance as $1 / r^{3}$. The field is symmetric relative to the plane $z=0$, due to it is even function of $z$. The lines of force are the essence of a circle. The energy of the potential component of the magnetic dipole (31) is calculated in the same way as that of the potential component of the electric charge:

$$
W_{\boldsymbol{B}}=\frac{1}{2 \mu} \iiint_{V_{\rho}}(\boldsymbol{B} \cdot \boldsymbol{B}) \mathrm{d} V=\frac{\mu p^{2}}{20 \pi \rho^{3}}
$$

In addition to the potential component of the magnetic field given by the relation (71), a magnetic field can have a non-potential component. To find it, one can use the technique described in Section 2. Let us take the auxiliary function in the form

$$
\begin{aligned}
\boldsymbol{H}^{+} & =\frac{z}{r^{5}}\{-y(1+\zeta), x, 0\} \\
& =\frac{1}{4 r^{2}}\left\langle-2 \zeta \sin 2 \varphi \sin ^{2} \vartheta,-\zeta \sin 2 \vartheta \sin 2 \varphi, 2(2+\zeta-\zeta \cos 2 \varphi) \sin \vartheta\right\rangle
\end{aligned}
$$

Here $\zeta$ is the constant which characterizes the deviation of the magnetic field from the potential one. At $\zeta=0$ the field is potential. The vector (73), i.e. the strength of the field produced by it, will be as follows

$$
\nabla \times \boldsymbol{H}^{+}=\frac{1}{r^{5}}\left\{3 x z, 3(1+\zeta) y z, x^{2}(\zeta-1)+z^{2}(2+\zeta)-y^{2}(1+2 \zeta)\right\}
$$

The divergence (73) will be as follows

$$
\nabla \cdot \boldsymbol{H}^{+}=\zeta \frac{3 \zeta \sin ^{2} \vartheta \sin 2 \varphi}{2 r^{3}}
$$

As neither (74) nor (75) are identically equal to zero, the vector (73) is neither potential nor rotational. As in the case of Section 2, it is necessary to find the potential vector $\Pi$ so that

$$
\nabla \cdot \Pi=\frac{3 \zeta \sin ^{2} \vartheta \sin 2 \varphi}{2 r^{3}}
$$

Then

$$
\nabla \cdot\left(\mathrm{H}^{+}-\Pi\right)=0
$$

Since the vector $\Pi$ is potential, it can be represented as the gradient of a 
certain function $\Psi$ :

$$
\Pi=\nabla \Psi
$$

Substituting this relation into (76) one obtains

$$
\Delta \Psi=\frac{3 \zeta \sin ^{2} \vartheta \sin 2 \varphi}{2 r^{3}}
$$

In the spherical coordinate system the equation is written as [8]:

$$
\frac{1}{r^{2}} \frac{\partial}{\partial r}\left(r^{2} \frac{\partial \Psi}{\partial r}\right)+\frac{1}{r^{2} \sin \vartheta} \frac{\partial}{\partial \vartheta}\left(\sin \vartheta \frac{\partial \Psi}{\partial \vartheta}\right)+\frac{1}{r^{2} \sin ^{2} \vartheta} \frac{\partial^{2} \Psi}{\partial^{2} \varphi^{2}}=\frac{3 \zeta \sin ^{2} \vartheta \sin 2 \varphi}{2 r^{3}}
$$

As in Section 0, let us assume that the unknown function $\Psi$ can be represented in the form of the product

$$
\Psi(r, \vartheta, \varphi)=R(r) \Theta(\vartheta) \Phi(\varphi)
$$

Assuming that $R(r)=1 / r$ one gets the equation

$$
\frac{\sin \vartheta}{\Theta(\vartheta)} \frac{\partial}{\partial \vartheta}\left(\sin \vartheta \frac{\partial \Theta(\vartheta)}{\partial \vartheta}\right)+\frac{1}{\Phi(\varphi)} \frac{\partial^{2} \Phi(\varphi)}{\partial \varphi^{2}}=\zeta \frac{3 \sin ^{4} \vartheta \sin 2 \varphi}{2 \Theta(\vartheta) \Phi(\varphi)}
$$

The left-hand side (82) is the sum of two terms, with the augend depending only on $\vartheta$, and the addend depending only on $\varphi$. The right-hand side (82) is the product of two unknown functions, one of them depending only on $\vartheta$, and the other only on $\varphi$. This is possible, when one of the terms is a constant value as well as the function corresponding to this term in the right-hand side (82). This assumption proves to be correct for the addend, and in this case, the value

$$
\frac{\sin 2 \varphi}{\Phi(\varphi)}=C_{0}
$$

Here the constant $\zeta$ is included into the arbitrary constant $C_{0}$, then

$$
\frac{1}{\Phi(\varphi)} \frac{\partial^{2} \Phi}{\partial \varphi^{2}}=-4
$$

and the Equation (82) goes into

$$
\sin \vartheta \frac{\partial}{\partial \vartheta}\left(\sin \vartheta \frac{\partial \Theta(\vartheta)}{\partial \vartheta}\right)-4 \Theta(\vartheta)=\frac{3}{2} C_{0} \sin ^{4} \vartheta
$$

Let us denote

$$
t=\ln \tan \frac{\vartheta}{2}
$$

therefore,

$$
\vartheta=2 \arctan \exp t
$$

Then using the differentiating rules

$$
\frac{\partial}{\partial \vartheta}=\frac{\partial}{\partial t} \frac{\partial t}{\partial \vartheta}=\frac{1}{\sin \vartheta} \frac{\partial}{\partial t}
$$

so 


$$
\sin \vartheta \frac{\partial}{\partial \vartheta}=\frac{\partial}{\partial t}
$$

and (85) goes into

$$
\frac{\partial^{2} \Theta}{\partial t^{2}}-4 \Theta=\frac{3 C_{0}}{2 \cosh ^{4} t}
$$

since one has the relations

$$
\sin \vartheta=\sin (2 \arctan \exp t)=2 \sin (\arctan \exp t) \cos (\arctan \exp t)=2 \frac{\mathrm{e}^{t}}{1+\mathrm{e}^{2 t}}
$$

The homogeneous equation for (90) will be written as

$$
\frac{\partial^{2} \Theta}{\partial t^{2}}-4 \Theta=0
$$

Its linearly independent solutions are

$$
\Theta_{1}=\sinh 2 \vartheta, \quad \Theta_{2}=\cosh 2 \vartheta
$$

Following the methods of solving such equations [8], let us consider the values

$$
\begin{gathered}
\frac{\mathrm{d} C_{1}}{\mathrm{~d} t}=\frac{3}{4} C_{0} \frac{\cosh 2 t}{\cosh ^{4} t}=\frac{3}{4} C_{0}\left(\frac{1}{\cosh ^{2} t}+\frac{\sinh ^{2} t}{\cosh ^{4} t}\right) \\
\frac{\mathrm{d} C_{2}}{\mathrm{~d} t}=-\frac{3}{4} C_{0} \frac{\sinh 2 t}{\cosh ^{4} t}=-\frac{3}{2} C_{0} \frac{\sinh t}{\cosh ^{3} t}
\end{gathered}
$$

Integrating them one obtains

$$
\begin{gathered}
C_{1}=\frac{C_{0}}{4}\left(\frac{1}{\cosh ^{2} t}-4\right) \\
C_{2}=\frac{3 C_{0}}{4 \cosh ^{2} t}
\end{gathered}
$$

The solution of the equation (90) will be written as

$$
\Theta=C_{0} \frac{2 \cosh 2 t+\cosh 4 t}{4 \cosh ^{2} t}+C_{3} \sinh 2 t+C_{4} \cosh 2 t
$$

Here $C_{3}, C_{4}$ are the arbitrary constants. Substituting there in (86), one derives

$$
\Theta=\frac{45 C_{0}+48 C_{4}-64 C_{3} \cos \vartheta+4\left(5 C_{0}+4 C_{4}\right) \cos 2 \vartheta-C_{0} \cos 4 \vartheta}{32 \sin ^{2} \vartheta}
$$

Substituting (95) into (85) one can make sure that (95) is the solution of this differential equation. Using (81) one derives the solution of the equation (80) in the form

$$
\Psi=\Theta \frac{\sin 2 \varphi}{r}
$$

Or turning to the Cartesian coordinates

$$
\begin{aligned}
\Psi= & \frac{x y}{2\left(x^{2}+y^{2}\right)^{2}\left(x^{2}+y^{2}+z^{2}\right)^{3 / 2}}\left\{C_{0}\left[3\left(x^{2}+y^{2}\right)^{2}+12\left(x^{2}+y^{2}\right) z^{2}+8 z^{4}\right]\right. \\
& \left.+4\left(x^{2}+y^{2}+z^{2}\right)\left[-2 C_{3} z \sqrt{x^{2}+y^{2}+z^{2}}+C_{4}\left(x^{2}+y^{2}+2 z^{2}\right)\right]\right\}
\end{aligned}
$$


Differentiating (97) one obtains

$$
\nabla \cdot \nabla \Psi=C_{0} \frac{3 \sin ^{2} \vartheta \sin 2 \varphi}{2 r^{3}}
$$

Hence it follows (75)

$$
C_{0}=\zeta
$$

Thus, one arbitrary constant in (97) has been defined. The vector

$$
\begin{aligned}
\nabla \Psi= & \frac{1}{32 r^{2} \sin ^{3} \vartheta}\left\langle\left[\zeta \cos 4 \vartheta-45 \zeta-48 C_{4}+64 C_{3} \cos \vartheta-4\left(5 \zeta+4 C_{4}\right) \cos 2 \vartheta\right] \sin \vartheta \sin 2 \varphi,\right. \\
& {\left[96 C_{3}-2\left(65 \zeta+64 C_{4}\right) \cos \vartheta+32 C_{3} \cos 2 \vartheta+3 \zeta \cos 3 \vartheta-\zeta \cos 5 \vartheta\right] \sin 2 \varphi, } \\
& {\left.\left[45 \zeta+48 C_{4}-64 C_{3} \cos \vartheta+4\left(\zeta+4 C_{4}\right) \cos 2 \vartheta-\zeta \cos 4 \vartheta\right] \cos 2 \varphi\right\rangle }
\end{aligned}
$$

is an unlimited value at $\vartheta \rightarrow 0$ at any distances from the origin of the coordinates, although its divergence is limited. In (100) the value of the vector $\nabla \Psi$ is given in the spherical coordinates. The total field vector

$$
\boldsymbol{H}^{+}+\nabla \Psi
$$

includes two arbitrary constants $C_{3}$ and $C_{4}$. The energy density

$$
w=\frac{\mu}{2}\left|\boldsymbol{H}^{+}+\nabla \Psi\right|^{2}
$$

produced by this vector field will be an unlimited value at $\vartheta \rightarrow 0$, therefore, the total energy

$$
W=\frac{\mu}{2} \int \mathrm{d} \vartheta \int_{0}^{2 \pi} \mathrm{d} \varphi \int_{\rho}^{\infty} w r^{2} \mathrm{~d} r
$$

will be unlimited too. During integration (103) first, let us find a certain integral with respect to $r$, then to $\varphi$, after that find some integral with respect to $\vartheta$, because a certain integral with respect to $\vartheta$ diverges at $\vartheta \rightarrow 0$, so as a result, one derives

$$
\begin{aligned}
W= & \frac{\pi}{3840 \rho}\left\{-30\left(192+128 C_{4}^{2}+192 \zeta+16 \zeta C_{4}-19 \zeta^{2}\right) \cos \vartheta\right. \\
& +2880 \zeta C_{3} \cos 2 \vartheta+5\left(125+32 \zeta\left(4+3 C_{4}\right)+173 \zeta^{2}\right) \cos 3 \vartheta \\
& -27 \zeta^{2} \cos 5 \vartheta+1920\left(C_{4}-C_{3}+\zeta\right)^{2}\left(\frac{1}{\sin ^{2} \frac{\vartheta}{2}}-\frac{1}{\sin ^{4} \frac{\vartheta}{2}}-4 \ln \sin \frac{\vartheta}{2}\right) \\
& \left.+1920\left(C_{4}+C_{3}+\zeta\right)^{2}\left(-\frac{1}{\cos ^{2} \frac{\vartheta}{2}}+\frac{1}{\cos ^{4} \frac{\vartheta}{2}}-4 \ln \cos \frac{\vartheta}{2}\right)\right\}
\end{aligned}
$$

In order to avoid the divergence with respect to $\vartheta$, one should use in (104):

$$
C_{4}-C_{3}+\zeta=0
$$




$$
C_{4}+C_{3}+\zeta=0
$$

Hence one finds

$$
\begin{gathered}
C_{3}=0 \\
C_{4}=-\zeta
\end{gathered}
$$

Substituting these values in (100) and (101) one obtains a non-potential vector in the form

$$
\begin{aligned}
\boldsymbol{H}^{+}+\nabla \Psi= & -\frac{1}{2 r^{5}}\left\{-y\left(\left(y^{2}+z^{2}\right)(2+\zeta)+2 x^{2}(1+2 \zeta)\right),\right. \\
& \left.x\left(-2 y^{2}(\zeta-1)+\left(x^{2}+z^{2}\right)(2+\zeta)\right),-3 \zeta x y z\right\} \\
= & \frac{1}{4 r^{2}}\left\langle 3 \zeta \sin 2 \varphi \sin ^{2} \vartheta, 0,-2(2+\zeta) \sin \vartheta\right\rangle
\end{aligned}
$$

Differentiating (105) with respect to the spatial coordinates in the Cartesian system of coordinates, one obtains multipoles of the corresponding order. For example, differentiating with respect to the coordinate $z$, one derives a magnetic dipole with the axis of symmetry along the $z$-axis in the form

$\frac{\partial}{\partial z}\left(\boldsymbol{H}^{+}+\nabla \Psi\right)=\frac{3 \mu}{4 \pi^{2} r^{3}}\left\langle-4 \zeta \cos \vartheta \sin ^{2} \vartheta \sin 2 \varphi,-\zeta \sin ^{3} \vartheta \sin 2 \varphi,(2+\zeta) \sin 2 \vartheta\right\rangle(106)$

This field is axially symmetric with the $z$-axis as the axis of symmetry, since the vector (106) is invariant with respect to the replacement $\varphi \rightarrow \varphi+\pi$. The azimuthal vector components (71), (106), (107) are proportional to one another, therefore, in the calculation of the interaction energy these components will yield a nonzero contribution to the interaction energy. This contribution, depending on the orientation of the neutron components can be both positive and negative. With the negative contribution the addition of the components (71) and (106) to (107) can decrease the total energy of the system, therefore, to correctly estimate the value of the magnetic moment of an elementary particle, it is necessary to take into consideration the influence of (71) and (106).

\section{Magnetic Moment}

For the electric field of the neutron $N$ (26) one has

$$
\nabla \times N=-\lambda_{n} \frac{3 e}{4 \pi \varepsilon r^{3}}\langle 0,0, \sin 2 \vartheta\rangle
$$

From here it is clear that in the spherical coordinate system only the azimuthal component of the vector (107) is other than zero. This field in its structure corresponds to the field of the potential magnetic dipole (71). For the correspondence to be complete, it necessary to multiply (107) by a certain value of $l_{d}$ having the dimensions of length. Then (107) gives the electric dipole field of the kind

$$
-\frac{D}{4 \pi \varepsilon r^{3}}\langle 0,0, \sin 2 \vartheta\rangle
$$


Here

$$
D=3 \lambda_{n} l_{d} e
$$

is the neutron dipole electric moment. Nowadays this value has not been measured yet, although there have been some attempts to do it [10] [11]. According to the Maxwell equation, there is interdependence between the rotor of the electric field strength and the vector of the magnetic field in the form

$$
\frac{\partial \boldsymbol{B}}{\partial t}=-\nabla \times \boldsymbol{N}+v \boldsymbol{B}
$$

Here $\boldsymbol{B}$ is the source of magnetic induction (71). In modern physics the sources of the magnetic field are not taken into account in the above equation, which is explained by the fact that monopoles have not been found yet. However, the magnetic dipole sources do exist, and when necessary they should be included into the Maxwell equation. Since a stationary case is considered, i.e. all values do not depend on time, then

$$
\frac{\partial \boldsymbol{B}}{\partial t}=0
$$

Hence it follows that

$$
v \boldsymbol{B}-\nabla \times \boldsymbol{N}=0
$$

The coefficient of proportionality $v$ has the dimensionality $\mathrm{s}^{-1}$. If (112) is multiplied by the coefficient with the dimensions of the length $l$, one will turn to the electric dipole (109) in the addend (112), and there will be the coefficient $l v$ having the dimensions of speed before the magnetic induction $\boldsymbol{B}$. Let us choose the value of $l$ so that $l v=c$, where $c$ is the light speed. Then one obtains the well-known relation in electrodynamics between the electric field strength and the magnetic induction $\boldsymbol{E}=c \boldsymbol{B}$. Comparing (71) and (107) one derives the expression for the electric dipole through the magnetic moment

$$
l_{n} e=\varepsilon c p
$$

Here it is denoted that $l_{n}=\lambda_{n} l$, which is the dipole moment. Substituting the value of the neutron magnetic moment $p=9.66237 \times 10^{-23} \mathrm{~A} \cdot \mathrm{s} \cdot \mathrm{cm}$ into (113) one obtains the value of the neutron electric dipole $l_{n}=2.5 \times 10^{-29} \mathrm{~cm}$.

Modern experimental methods [10] [11] do not possess the required resolution to measure the above value of the neutron electric moment, so the question of its existence remains open. Thus, the non-potential electric field gives rise to the magnetic dipole moment of elementary particles. This magnetic dipole possesses nonzero energy of interaction with the dipole sources of the magnetic field (71) and (106). The total magnetic dipole moment will be their linear combination. Determination of the coefficients of this linear combination is outside the scope of the present paper.

\section{Conclusion}

As shown above, if one takes into consideration the non-potential electric field, 
then the known particles prove to have properties unknown earlier and the known properties find a simple explanation. It is common knowledge that scattering of elementary particles has the property of indefiniteness, which is explained by a special character of the microworld having no analogy in the macroscopic world. Taking into account the non-potential components of the electric field eliminates the "mysteriousness". Orientation of the neutron components of the colliding particles is arbitrary, uncontrolled at the moment, and the interaction force depends on it. Therefore, the result of scattering of the particles will have a fraction of indefiniteness. Another source of indefiniteness is the fact that the neutron component is proportional to the parameter $\lambda_{n}$. When particles do not interact, the neutron component is in the stationary state, at which the value of $\lambda_{n}$ is determined by the source. If the interaction is weak, then after it the orientation of the neutron components of the particles will not change. This is elastic scattering. However, if the interaction is strong, then the parameter $\lambda_{n}$ of the neutron components may increase during collision increasing the neutron component, and the particles go into the excited state, in which the neutron components get a nonstationary addition and there appear electromagnetic oscillations. The orientation of the neutron components after the collision may change and result in radiation. This is nonelastic scattering.

Another characteristic of nonpotentiality is the existence of the proton neutron, electron-neutron and neutron-neutron components of interaction. Neutrons and protons have similar properties and for this reason, they belong to the same group of particles-nucleons. In terms of the potential theory, it is not at all clear. Neutrons have no charge and they have "no" electric field, so they must not interact electrostatically with a proton. However, the interaction takes place even at small energies, for example, in the case of cold neutrons [9]. There is no explanation for the fact. Thus, in the potential theory the interaction of a neutron with a proton is a puzzle. For the non-potential field there is no problem like this. The energy of interaction between a neutron and a proton is derived by taking the sum of (60) and (65)

$$
\begin{aligned}
W_{p n}= & W_{e n}+W_{n n}=\frac{\lambda_{n} e^{2}}{1536 \pi \varepsilon a}\left[-6\left(16 Q-9+18 \lambda_{n}\right) \cos 2 \beta \cos ^{2} \gamma\right. \\
& +18 \lambda_{n} \cos 2 \beta_{-}\left(3+10 \cos 2 \beta \cos ^{2} \gamma-9 \cos 2 \gamma\right) \\
& \left.+\left(16 Q-9-6 \lambda_{n}\right)(3 \cos 2 \gamma-1)-576 \lambda_{n} \cos ^{2} \gamma \sin 2 \beta \sin 2 \beta_{-}\right]
\end{aligned}
$$

The energy of interaction of a proton with a proton is given by the relation (67). When comparing (67) and (114) one can see that the coefficient in front of the square brackets of both formulas, as can be checked by substitution of various angle values and for $\lambda_{n}=1$, is of the same order of magnitude. For instance, for $\beta=\beta_{-}=\gamma=0$ one obtains for the proton-proton interaction (67)

$$
W=\frac{e^{2}\left[24+\left(9-16 Q-6 \lambda_{n}\right) \lambda_{n}\right]}{192 \pi \varepsilon a}
$$


and the energy of the proton-neutron interaction (114) is written as

$$
W_{p n}=\frac{\lambda_{n} e^{2}(9-16 Q)}{384 \pi \varepsilon a}
$$

Using the relation (58) one derives that for $\lambda_{n} \sim 1$ the values of (115) and (116) are of the same order of magnitude. Therefore, due to the electric field, the interaction between a neutron and a proton is approximately the same as that between a proton and proton. That explains the similarity of these particles. In terms of the potential theory, the existence of an antineutron and its annihilation with a proton are not clear either. What is the difference between a neutron and an antineutron? In the light of the non-potential theory, these are the natural consequences of the properties of the intrinsic electric fields of the particles. Choosing the corresponding sign before $\lambda_{n}$, one obtains the negative interaction energy (114). The electric field non-potentiality results in the elementary particles acquiring the magnetic moment. The particles also have the electric dipole moment, which has not been measured so far due to the insufficient experiment accuracy.

\section{References}

[1] Vladimirov, V.S. (1981) Equations of Mathematical Physics. Nauka, Moscow.

[2] Lavrent'ev, M.A. and Shabat, B.V. (1973) Methods of Complex Variable Theory. Nauka, Moscow.

[3] Ivanchin, A.G. (1987) An Application of Potential Theory for Solving the Plane Elastic Crack Problem. In: Goerlich, P., Ed., Physica Status Solidi (a), Akademie Verlag Berlin, Berlin, 437-447. https://doi.org/10.1002/pssa.2210990213

[4] Ivanchin, A.G. (2011) Nonpotential Solution of the Electon Problem. https://arxiv.org/ftp/arxiv/papers/0902/0902.1286.pdf

[5] Ivanchin, A.G. (2010) Potential Solution of Poisson's Equation. https://arxiv.org/ftp/arxiv/papers/1011/1011.4723.pdf

[6] Landau, L.D. and Lifshitz, E.M. (1995) The Classical Theory of Fields. Pergamon Press, Oxford.

[7] Wolfram Mathematica Online. http://www.wolfram.com/mathematica/

[8] Korn, G.A. and Korn, T.M. (1968) Mathematical Handbook. McGraw-Hill Book Company, London.

[9] Gurevich, I.I. and Tarasov, L.V. (1965) Physics of Low-Energy Neutrons. Nauka, Moscow.

[10] Serebrov, A.P., et al. (1983) New Measurements of the Neutron Electric Dipole Moment with the Petersburg Nuclear Physics Institute Double Chamber Electric Dipole Moment Spectrometer. Physics of Particles and Nuclei Letters, 12, 286-296. http://www.physics.princeton.edu/ kirkmcd/examples/EP/serebrov_ppnl_12_286_ 15.pdf

[11] Shabalin, Y.P. (1983) The Neutron Electric Dipole Moment in the Calibration Theory. In: Ginzburg, V.L., Ed., Achievements in Physical Science, vol. 139. 